\title{
Clinical characteristics and course of plantar vein thrombosis: A series of 22 cases
}

Phlebology

2015, Vol. 30(10) 714-718

(C) The Author(s) 2014

Reprints and permissions: sagepub.co.uk/journalsPermissions.nav DOI: 10.1 177/02683555|4555385 phl.sagepub.com

@SAGE

\author{
M Czihal', J Röling' , A Rademacher ${ }^{3}$, A Schröttle', \\ P Kuhlencordt' and U Hoffmann'
}

\begin{abstract}
Objectives: To evaluate the clinical presentation and disease course of symptomatic plantar vein thrombosis. Patients and methods: Patients with a first diagnosis of symptomatic plantar vein thrombosis at our institution were retrospectively identified from a prospectively maintained database. All patients underwent complete venous compression sonography extended to the plantar veins because of local symptoms at the sole of the foot. Clinical characteristics were obtained from the medical records.

Results: Between 2005 and 2013, 22 patients were diagnosed with a first episode of plantar vein thrombosis (64\% women, mean age at diagnosis 58.2 years, range 32-79 years). All patients reported moderate to heavy pain of the sole of the foot. The lateral plantar veins $(96 \%)$ were more frequently affected than the medial plantar veins $(41 \%)$ and extension into the deep calf veins was common (27\%). Half of the episodes were idiopathic, with subsequent diagnosis of occult malignancy in two of these patients. In seven patients (32\%), plantar vein thrombosis occurred in association to physical strain to the foot. All patients were treated with anticoagulation. Symptomatic pulmonary embolism was not observed and during a mean follow up of 21 months, the post-thrombotic syndrome did not occur. However, recurrences were common (27\%) and frequently again affected the plantar veins.

Conclusion: Plantar vein thrombosis should be considered as an important differential diagnosis of acute foot pain.
\end{abstract}

\section{Keywords}

Anatomy, anticoagulation, deep vein thrombosis, venous disease, venous thromboembolism

\section{Introduction}

Plantar vein thrombosis (PVT) is rarely considered as a differential diagnosis of foot pain. Moreover, the plantar veins are not routinely assessed in current compression sonography protocols for the diagnosis of deep venous thrombosis. Less than 30 cases of PVT have been reported in the medical literature during the last two decades. ${ }^{1-3}$

The aim of the present study was to evaluate the clinical and imaging characteristics as well as the outcome under anticoagulant treatment in a consecutive single-center series of patients with symptomatic PVT.

\section{Patients and methods}

Patients with a first diagnosis of symptomatic PVT at our institution were retrospectively identified from a prospectively maintained database.
All patients had undergone complete venous compression sonography of the symptomatic leg at baseline, with the examination extended to the calcaneal confluent at the level of the ankle and the medial and lateral plantar veins because of local symptoms at the sole of the foot. PVT was diagnosed if compression sonography revealed noncompressible medial and/or lateral plantar veins secondary to intraluminal echogenic material (Figure 1). ${ }^{4}$ Proximal extension into the posterior tibial veins as well as concomitant superficial thrombophlebitis were documented.

\footnotetext{
'Division of Vascular Medicine, Munich University Hospital, Germany ${ }^{2}$ Department of Internal Medicine II, Klinikum Augsburg, Germany ${ }^{3}$ Internistisches Zentrum, Poing, Germany

Corresponding author:

Michael Czihal, Division of Vascular Medicine, Medical Clinic and Policlinic IV, Munich University Hospital, Pettenkoferstrasse 8a, D-80336 Munich, Germany.

Email: michael.czihal@med.uni-muenchen.de
} 


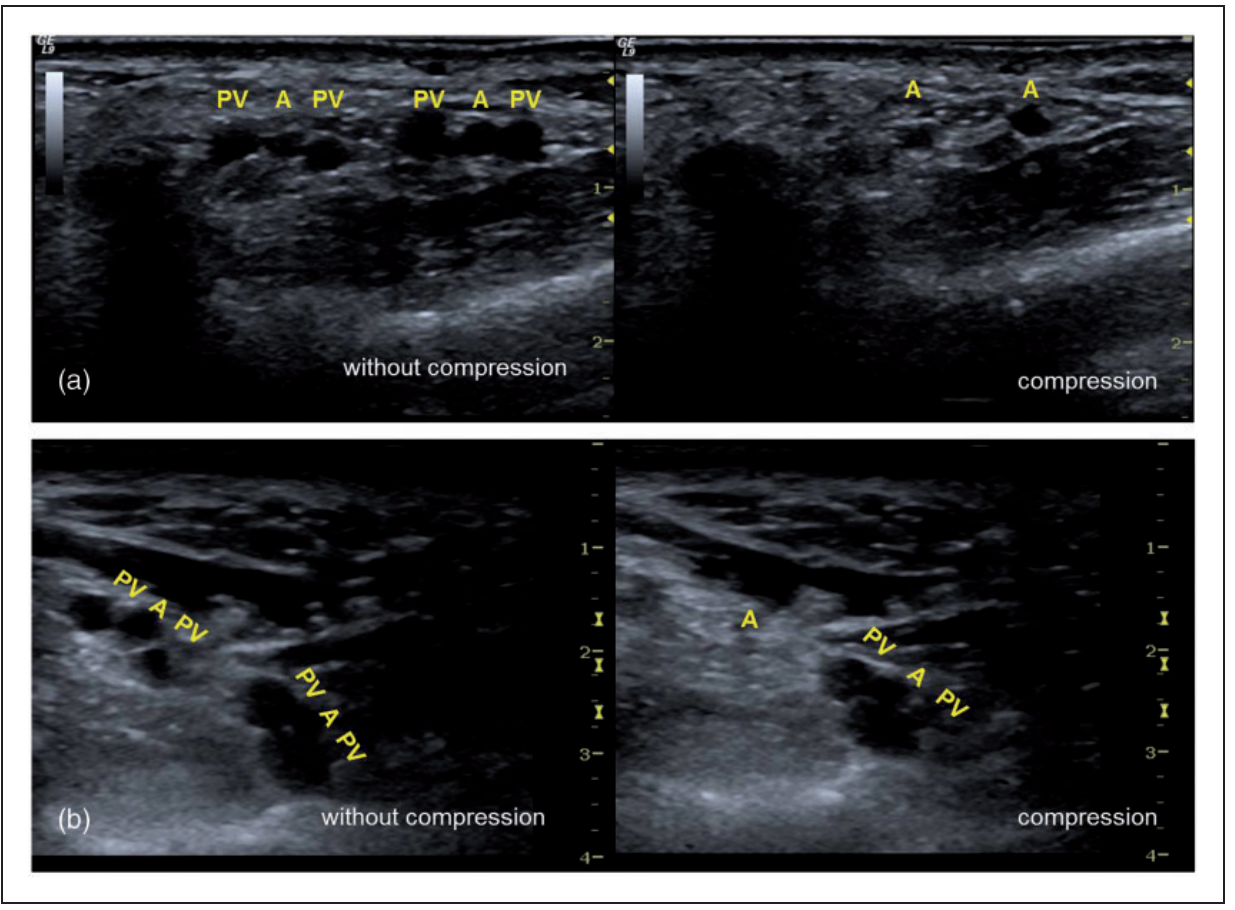

Figure I. Compression sonography of the PV and the adjacent arteries (A): (a) complete compressibility of the medial and lateral plantar veins in a healthy person and (b) a patient with acute symptomatic PVT: the lateral plantar veins are not compressible. PVT: plantar vein thrombosis; PV: plantar veins.

Clinical characteristics including risk factors and symptoms, imaging data, treatment modalities and follow-up information were obtained from the medical records.

\section{Results}

\section{Clinical characteristics}

Between 2005 and 2013, 22 patients were diagnosed with a first episode of PVT at our institution $(63.6 \%$ women, mean age at diagnosis 58.2 years, range $32-79$ years).

The left and right foot was affected in 12 and 10 patients, respectively. All patients reported moderate to heavy pain at the sole of the foot and seven patients additionally complained of pain at the medial malleolus. Swelling/edema of the foot and the ankle was present in seven and six patients, respectively. In three patients, superficial thrombophlebitis was clinically obvious. Clinical symptoms suggesting pulmonary embolism were not present in any of the patients at the time of diagnosis.

In seven patients (five women), development of foot pain coincided to mechanical strain to the affected foot in terms of new insoles (two patients), new high heels (one patient), exhaustive walking (three patients) or standing with bare feet on a ladder while working (one patient). The case of a 32-year-old female suffering from PVT after a busy night duty on intensive care unit has been published previously. ${ }^{5}$ Two post-menopausal women were on hormone replacement therapy and two female patients used hormonal contraceptives when PVT occurred. Three out of four episodes in women taking sexual hormones occurred in association to mechanical strain to the foot. Immobilization as a thrombosis risk factor was present in only two patients, i.e. a 79-year-old woman who had undergone permanent pace maker implantation and a 48-year-old woman who developed foot pain after a $6 \mathrm{~h}$ train ride. One patient experienced PVT during a disease flare of ulcerative colitis.

In $50 \%$ of our patients, no clinically obvious cause for the thrombotic event could be identified. Four of these patients had a history of prior deep venous thrombosis and one patient had a history of prior superficial thrombophlebitis.

Screening for hereditary and acquired thrombophilia was performed in 14 of 22 patients (63.6\%). Heterozygote mutation of the MTHFR gene, the prothrombin gene and the Factor $\mathrm{V}$ gene was found in 5, 1 and 1 patients, respectively. Other hereditary or acquired prothrombotic states were not found in any of the patients tested. Findings of thrombophilia screening did not modify treatment decisions in any of our patients tested. 


\section{Diagnosis}

The majority of patients primarily contacted the general physician (15 patients). Noteworthy, an orthopedic specialist was consulted in eight patients prior to referral to our department. The mean time between symptom onset and diagnosis was 8.8 days (range 1-21 days).

Five patients had undergone a magnetic resonance imaging study of the foot. Complete compression sonography (Figure 1) revealed involvement of the lateral plantar veins in almost all cases $(95.5 \%)$. In contrast, thrombosis of the medial plantar veins was documented in only $40.9 \%$ of cases, with isolated thrombosis of the medial plantar veins seen in just one patient. Involvement of both the lateral and medial plantar veins was present in six patients $(36.6 \%)$.

In 6 patients, thrombosis was confined to the plantar veins only, whereas thrombosis extended into the calcaneal confluent at the level of the medial malleolus (10 cases) or the posterior tibial veins (6 cases). One 79-year-old female patient with PVT extending into the calcaneal confluent also had calf muscle vein thrombosis. Frequently ( 7 out of 22 patients), superficial thrombophlebitis of the foot or lower leg occurred concomitantly. In a 68 -year-old male patient who was subsequently diagnosed with metastatic lung cancer (see below), PVT extended into the tibial posterior veins and migratory superficial thrombophlebitis of the ipsilateral great and short saphenous vein as well as the contralateral cephalic vein was present.

\section{Treatment and outcome}

The mean follow-up period was 21.2 months (range 1-71 months). All patients were treated with kneelength compression stockings. All but one patient initially received low-molecular weight heparin, unfractionated heparin or fondaparinux in full therapeutic doses. One patient with thrombosis confined to the plantar veins was treated with low-molecular weight heparin in a prophylactic dose. In general, patients with the thrombus restricted to the plantar veins or the calcaneal confluent and no persistent risk factor were treated with low-molecular weight heparin or fondaparinux for 4-6 weeks. Patients with thrombus extension into the deep veins above the ankle but no persistent risk factor were treated according to current guidelines for 3 months with low-molecular weight heparin overlapping with vitamin $\mathrm{K}$ antagonists. Except for mild oral mucosal bleeding occurring in a 69-year-old female under vitamin $\mathrm{K}$ antagonist treatment, no bleeding complications were observed.

Idiopathic PVT was associated with occult malignancy in two patients (non-small cell lung cancer, breast cancer recurrence) and with monoclonal gammopathy of unknown significance in another patient. The patient suffering from lung cancer developed recurrent pulmonary embolism during effective anticoagulant treatment and finally underwent implantation of an inferior vena cava filter. He died 2 years after diagnosis, secondary to bacterial sepsis during palliative chemotherapy. None of the remaining patients developed symptomatic recurrent venous thromboembolism under anticoagulant treatment.

In the patient with active breast cancer recurrence, we recommended to continue anticoagulant treatment indefinitely. However, the patient stopped treatment after 3 months. Subsequently ( 5 months after diagnosis of PVT), she developed pulmonary embolism and fibular vein thrombosis of the contralateral leg was diagnosed. A male patient who initially (at the age of 68 years) suffered from isolated PVT in association with physical strain to the foot developed recurrent ipsilateral PVT without any identifiable provoking factor 2 years after treatment cessation and finally was diagnosed with idiopathic thrombosis of the ispilateral posterior tibial veins 5 years after the initial event. Another three patients (one patient with PVT in association with physical strain to the foot and two patients with idiopathic PVT) experienced recurrent PVT after stopping anticoagulation. The recurrent event affected the ipsiand contralateral foot in one and two cases, respectively.

Clinical symptoms of PVT resolved after a mean time of 15.9 days (range 4-42 days). Repeat ultrasound of the plantar veins was performed in 17 of 22 patients after a mean follow-up of 22.6 months, showing residual thrombus and plantar vein insufficiency in 11 and 6 patients, respectively. During the follow-up period, none of the patients developed signs or symptoms of post-thrombotic syndrome.

\section{Discussion}

Herein, we report the largest series of patients diagnosed with symptomatic PVT thus far. Our main findings can be summarized as follows:

(1) Moderate to heavy foot pain is the pivotal clinical symptom of PVT.

(2) The lateral plantar veins are affected in almost all cases and extension into the deep and/or superficial venous system of the lower leg is common.

(3) Up to $50 \%$ of episodes are idiopathic and idiopathic PVT may herald occult malignancy in some cases.

(4) Physical strain to the sole of the foot may be considered an important risk factor for PVT.

(5) Anticoagulant treatment for 4-6 weeks appears to be a reasonable treatment option in the management of isolated PVT. 
(6) Recurrent venous thromboembolism is not uncommon (27\% in our series) and frequently manifests again as PVT.

Remarkably, historic venographic studies in patients with suspected venous thromboembolism revealed the prevalence of vein thrombosis of the foot in as much as $31 \%$, with isolated thrombosis below the ankle being present in $10 \%$ of venograms. ${ }^{6}$ These findings contrast with the paucity of reports on symptomatic PVT, with less than 30 cases published in the last 20 years. ${ }^{1-3}$ Indeed, PVT may be frequently misdiagnosed in the clinical setting of acute foot pain. It is noteworthy that a considerable proportion of our patients primarily contacted an orthopedic specialist and/or underwent magnetic resonance imaging of the foot for a suspected musculoskeletal cause of foot pain. Besides musculoskeletal disorders such as plantar fasciitis or stress fractures of the metatarsal bones, PVT should be included into the differential diagnosis of acute unilateral foot pain. Diagnosis is quickly established by compression sonography. The investigation is not costly and appropriate sonography devices are widely available.

From the deep plantar arch, the medial and lateral plantar veins run backwards adjacent to the corresponding arteries. After communicating with the great and small saphenous veins, they form the calcaneal confluent of the posterior tibial veins behind the medial malleolus. ${ }^{1,4}$ While the medial plantar pedicle is short and located between tendons, the lateral plantar pedicle is longer, larger and located between muscle layers. Thus, from a functional perspective, the lateral plantar veins represent the main component of the plantar venous pump. ${ }^{7}$ Our series indicates that the lateral plantar veins are also predominantly affected by PVT.

Of note, physical strain to the sole of the foot could be a possible risk factor for PVT. Excessive mechanical stress to the plantar veins during exercise may lead to repeated microtrauma of the vessel intima with consecutive activation of the coagulation cascade and development of PVT. In our cohort, this potential risk factor was documented in $32 \%$ of patients. Whether this is actually a causal association cannot be determined on the basis of this case series.

In contrast, half of the episodes observed were nonprovoked, idiopathic episodes, with two of these patients being diagnosed with occult malignancy. Thus, it may be worthwhile to screen for occult malignancy in PVT patients without other identifiable risk factors for thrombosis. The deep calf veins are thought to be the most common origin for the development of proximal deep venous thrombosis. ${ }^{8}$ Whether untreated
PVT also may be a potential starting point of an ascending deep venous thrombosis involving the more proximal veins remains unclear. Limited data suggests that withholding anticoagulation and treatment with non-steroidal anti-inflammatory drugs only may be associated with a risk of progression of PVT into the deep calf veins. ${ }^{1,4}$ Anticoagulation for a duration of 4-6 weeks for isolated PVT and for 3 months in case of extension into the deep calf veins appeared to be safe and effective in our series. However, as of today, there is insufficient data to give clear recommendations on duration and intensity (prophylactic versus therapeutic) of anticoagulation in PVT.

Symptomatic pulmonary embolism is a very rare event in patients with PVT. ${ }^{1,2}$ Moreover, signs and symptoms attributable to a post-thrombotic syndrome were not observed in our patients after a mean follow-up of 21 months despite residual thrombus and insufficiency of the plantar veins in the majority of the patients. Recurrent symptomatic PVT may occur after stopping anticoagulation and usually again affects the ipsi- or contralateral plantar veins.

In conclusion, PVT shares common risk factors with more proximal deep venous thrombosis and therefore seems to be the minimal variant of a common disease in most patients. However, mechanical strain to the foot appears to be a unique risk factor for PVT, which in these cases may be considered as a distinct variant of venous thromboembolic disease. In accordance with current concepts of distal venous thromboembolism, PVT carries a very low risk of (symptomatic) pulmonary embolism and the post-thrombotic syndrome.

\section{Conflict of interest}

None declared.

\section{Funding}

This research received no specific grant from any funding agency in the public, commercial, or not-for-profit sectors.

\section{References}

1. Karam L, Tabet G, Nakad J, et al. Spontaneous plantar vein thrombosis: state of the art. Phlebology 2013; 28: 432-437.

2. Barros M, Nascimento I, Barros T, et al. Plantar vein thrombosis and pulmonary embolism. Phlebology 2015; 30: 66-69.

3. Bruetman JE, Andrews JA, Finn BC, et al. Plantar vein thrombosis as a cause of local pain. Medicina ( $B$ Aires) 2014; 74: 87-88.

4. Barros MV and Labropoulos N. Plantar vein thrombosisevaluation by ultrasound and clinical outcome. Angiology 2010; 61: 82-85. 
5. Geiger C, Rademacher A, Chappell D, et al. Plantar vein thrombosis due to busy night duty on intensive care unit. Clin Appl Thromb Hemost 2011; 17: 232-234.

6. Thomas ML and O'Dwyer JA. A phlebographic study of the incidence and significance of venous thrombosis in the foot. Am J Roentgenol 1978; 130: 751-754.
7. Uhl JF and Gillot C. Anatomy of the foot venous pump: physiology and influence on chronic venous disease. Phlebology 2012; 27: 219-230.

8. Palareti $G$ and Schellong S. Isolated distal deep vein thrombosis: what we know and what we are doing. J Thromb Haemost 2012; 10: 11-19. 